\title{
Research Paper: Perceptions of the Parents of Children With Autism Spectrum Disorders and Healthcare Providers on the Challenges of Using Rehabilitation Services in Iran
}

\author{
Mahta Alsadat Aarabi' ${ }^{1}$ (D), Kianoush Abdi ${ }^{*}$ (D), Mohammad Saeed Khanjani² (D) \\ 1. Department of Rehabilitation Management, University of Social Welfare and Rehabilitation Sciences, Tehran, Iran. \\ 2. Department of Counseling, University of Social Welfare and Rehabilitation Sciences, Tehran, Iran.
}

\begin{tabular}{|c|c|}
\hline $\begin{array}{l}\text { Use your device to scan } \\
\text { and read the article online }\end{array}$ & Cittation: Aarabi MS, Abdi K, Khanjani MS. Perceptions of the Parents of Children With Autism Spectrum Disorders and \\
\hline aristis & $\begin{array}{l}\text { Healthcare Providers on the Challenges of Using Rehabilitation Services in Iran. Iranian Rehabilitation Journal. 2021; 19(1):13- } \\
\text { 22. http://dx.doi.org/10.32598/irj.19.1.1234.3 }\end{array}$ \\
\hline abitas & dol'http://dx.doi.org/10.32598/irj.19.1.1234.3 \\
\hline
\end{tabular}

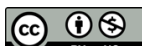

Article info:

Received: 07 Apr 2021

Accepted: 20 Apr 2021

Available Online: 01 Mar 2021

Keywords:

Autism Spectrum Disorder (ASD), Challenge, Rehabilitation services, Healthcare, Qualitative

\section{ABSTRACT}

Objectives: One of the most essential needs of children with Autism Spectrum Disorder (ASD) is using rehabilitation services. However, these services are associated with numerous challenges. The present study aimed to identify the perceptions of the parents of children with ASD and rehabilitation service providers about the challenges of using rehabilitation services in Iran.

Methods: This was a qualitative study. In total, 27 participants were selected from the parents of children with ASD and rehabilitation service providers to these children, based on purposive sampling method; we considered maximum variation and sampling continued until data saturation. The necessary data were collected by semi-structured and in-depth interviews. The interviews lasted from July to December 2020 and MAXQDA was used for data analysis.

Results: The challenges of children with ASD in accessing and using rehabilitation services were identified and categorized into 6 main classes, as follows: the lack of a systematic support system; the lack of commitment to observing professional ethics; neglecting the structure and policy of rehabilitation; the lack of comprehensive ASD diagnostic and rehabilitation protocols; inadequate knowledge and attitude, and the lack of feasible access to services.

Discussion: Policymakers, service providers, and managers must be aware of the challenges of rehabilitation services for ASD; subsequently, they are expected to pay special attention to this issue. This study provided evidence for the above-mentioned groups for accessing and using rehabilitation services among ASD. Besides, the obtained data could help to improve the services they receive and their living conditions; ultimately, they help improve their quality of life. 


\section{Highlights}

- This research has shown that there are serious problems in using and accessing rehabilitation services for children with ASD and their families.

- Rehabilitation services require serious long-term and short-term planning, as well as coordination between autism organizations and stakeholders. This can improve their living conditions and ultimately increase their quality of life.

- Our results provide a clear perspective for policymakers, managers and planners to change their perspective and consider ASD as a key part of rehabilitation programs to reform executive structures.

\section{Plain Language Summary}

This study was conducted to find the exact challenges of accessing and using rehabilitation services for children with autism spectrum disorder and their families. The results of this study were obtained through interviews with 27 parents of children with autism and rehabilitation service providers. The findings of this study divide these challenges into the following six groups: 1) Lack of a systematic support system 2) No commitment to the observance of professional ethics 3) Neglecting the structure and policy of ASD rehabilitation 4) Lack of comprehensive autism diagnostic and rehabilitation protocols 5) Lack of knowledge and proper attitude 6) Barriers to easy access to rehabilitation services.

\section{Introduction}

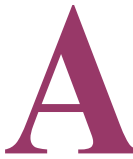

utism Spectrum Disorder (ASD) is a complex neurodevelopmental condition. ASD is characterized by a lack of social communication. Furthermore, very limited interests and repetitive behaviors are its prominent features [1-3] The prevalence of ASD is 1 in 54 children [4]. The fastest growth rate of any disability belongs to ASD since 2009 , with an annual increase of $10.9 \%$ [5]. Most children with ASD encounter significant problems, such as social interaction and communication skills. Thus, they should receive some type of rehabilitation training to improve their socio-communication skills, behavioral defects, and sensory problems [6]. Children with ASD, according to their specific characteristics and conditions, require a special intervention to modify and eliminate the symptoms of the disorder and improve their living conditions. Various interventions, such as medical approaches, behavioral analysis based on parental education, etc. can greatly help improve these children's conditions [7].

Ensuring access to healthcare and rehabilitation systems is critical for the proper diagnosis and interventions in children with ASD [8]. However, access to such healthcare services, as rehabilitation seems to be challenging for this population [9-12]. Furthermore, healthcare providers are not well prepared to provide appropriate services to such patients. For example, they lack sufficient skills in some aspects of the rehabilitation services. Additionally, they reported a lack of skill and equipment, i.e. required for ASD management [13]. Interventions in individuals with ASD require applying appropriate facilities and structures, such as physical space; human resources; financial resources and equipment; professional support, and the cooperation of children's families, especially parents [14]. Given the special circumstances and needs of children with ASD, an urgent necessity is accessing rehabilitation services. Such measures could help to improve their performance in the required dimensions.

Considering the relevant studies as well as the recent changes in various ASD centers, multiple intervention protocols have been developed and employed. This qualitative study aimed to determine the challenges of children with ASD to use rehabilitation services. We also addressed the lack of knowledge using a qualitative approach, complexity of the studied phenomenon, and dependence on socio-cultural factors in the context and environment they live in. Besides, studies based on the experiences of parents and therapists are scarce.

\section{Methods}

The present qualitative research used conventional content analysis. We aimed to identify the challenges of using rehabilitation services for children with ASD. Researchers generally use the conventional content analysis method in studies aimed at describing a phenomenon without a theoretical framework and prede- 
termined categories [15]. In this method, concepts are directly extracted from the data by individual interviews and post coding. Qualitative content analysis is applied to interpret the content of textual data that can be identified through a systematic classification process, explicit themes, or patterns in the text [16].

Twenty-seven participants were selected using purposive sampling and the maximum variation method. Accordingly, data gathering was continued until data saturation, i.e. until no additional data were found. The study participants included 14 therapists (rehabilitation service providers) and 13 parents (the recipients of rehabilitation services) of children with ASD in Tehran City, Iran. The research participants were selected from the parents of different genders with children with ASD at different ages; educational and cultural levels; as well as different socioeconomic conditions, and religious status. The explored therapists were selected from various fields of rehabilitation, including occupational therapy and speech therapy.

The inclusion criteria of the study were as follows: willingness to participate in the study; parents with children aged 3-15 years; parents of children with ASD who have received continuous rehabilitation services for at least one year; and therapists with $\geq 3$ years of experience in providing rehabilitation services for children with ASDs.

To collect the required data, we used in-depth and semi-structured interviews with open-ended questions; if necessary, follow-up and probing questions were employed. Initially, the interview began with open-ended questions. In the subsequent interviews, the questions were arranged based on the extracted categories. For example, starting with the following question and other questions were adjusted based on the respondent's answer: (for parents) "Can you tell us about your problems with using rehabilitation services for your children?" and (for therapists) "Can you tell us about the problems you encountered in providing rehabilitation services to children with ASD?" Follow-up or probing questions were used based on the study participants' answers. Before conducting the interview, the study purposes were explained to the research participants. Moreover, they provided an informed consent form. The interviews were performed from July 2020 to December 2020 and lasted between 20 and 60 minutes. They were recorded and transcribed verbatim.

To analyze the obtained qualitative data, the conventional content analysis method of Granheim and Lundman (2004) was used [17]. This approach consists of 3 stages of preparation, organization, and reporting. In the preparation, an analysis unit that could be a sentence was selected [18]. The organization stage consists of 3 steps: open coding, classification, and abstraction. In the organization phase, the prior categories and classes were not used; instead, the researcher immersed himself in the data and attempted to form new perspectives and obtain new categories from the collected data. Then, he chose new names for these new groupings that contained the themes of that group. In the open coding phase, each text was read several times; then, it was analyzed to better understand the study participants' experiences and feelings. In the classification stage, the codes were classified. The purpose of data grouping was to reduce the number of categories by merging similar classes into larger groups. The final step of the content analysis approach was to report the achieved results. In this step, we provided examples for each code and category [16]. Moreover, data analysis was conducted using MAXQDA.

To observe the reliability of the obtained qualitative data, we used 4 criteria of credibility, transferability, dependability, and confirmability. This classification was proposed by Guba and Lincoln. It is among the most commonly used criteria for evaluating the quality of qualitative data $[19,20]$.

Furthermore, the ethical principles of keeping information confidential and obtaining informed consent from participants before the interview for interviewing and recording them; as well as considering the right for participants to discontinue participation at any stage of the study were observed. This study was extracted from a Master's thesis approved by the Ethics Committee of the University of Social Welfare and Rehabilitation Sciences (Code: IR.USWR.REC.1399.144).

\section{Results}

The study participants included 27 subjects whose demographic characteristics are mentioned in Table 1 . The results of data analysis in the form of 6 categories (main concepts) and 25 sub-categories, as the challenges of rehabilitation services for children with ASD, are presented in Table 2.

\section{The challenges of rehabilitation services for ASD}

\section{The lack of a systematic support system}

The present study findings revealed that an essential challenge, based on participants' experiences, was the lack of systematic support; this class had three subcategories, as follows: the lack of insurance coverage; 
Table 1. The demographic characteristics of the research participants

\begin{tabular}{|c|c|c|c|c|}
\hline Code & Gender & Education & Type & Experience $(y)$ \\
\hline 1 & Male & BSC & Speech therapy & 20 \\
\hline 2 & Male & MSc & Speech therapy & 4 \\
\hline 3 & Female & BSc & Parent & 4 \\
\hline 4 & Female & $\mathrm{PhD}$ & Speech therapy & 19 \\
\hline 5 & Female & BSc & Parent & 3 \\
\hline 6 & Female & MSc & Speech therapy & 7 \\
\hline 7 & Female & AS & Parent & 5 \\
\hline 8 & Male & BSc & Speech therapy & 18 \\
\hline 9 & Female & BSc & Occupational therapy & 3 \\
\hline 10 & Male & $\mathrm{PhD}$ & Occupational therapy \& Neuroscience & 20 \\
\hline 11 & Male & $\mathrm{PhD}$ & Occupational therapy \& Psychology \& Psychotherapy & 23 \\
\hline 12 & Female & MSc & Speech therapy & 23 \\
\hline 13 & Male & $\mathrm{PhD}$ & Occupational therapy & 24 \\
\hline 14 & Male & $\mathrm{PhD}$ & Occupational therapy \& Cognitive neuroscience & 23 \\
\hline 15 & Female & BA & Parent & 6 \\
\hline 16 & Female & $\mathrm{BSC}$ & Parent & 3.5 \\
\hline 17 & Female & BSc & Parent & 3 \\
\hline 18 & Male & AS & Parent & 3 \\
\hline 19 & Female & $\begin{array}{l}\text { Primary educa- } \\
\text { tion }\end{array}$ & Parent & 6 \\
\hline 20 & Female & BSc & Parent & 5 \\
\hline 21 & Female & AS & Parent & 4 \\
\hline 22 & Female & AS & Preschool & 3 \\
\hline 23 & Female & $\mathrm{BSc}$ & Parent & 3.5 \\
\hline 24 & Female & AS & Parent & 4 \\
\hline 25 & Male & $\mathrm{PhD}$ & Speech therapy \& Health psychology & 29 \\
\hline 26 & Female & MSc & Occupational therapy \& Rehabilitation management & 11 \\
\hline 27 & Female & $\mathrm{BSC}$ & Parent & 7 \\
\hline
\end{tabular}


Table 2. The challenges of rehabilitation services for children with ASD from the study participants' perspective

\begin{tabular}{ll}
\hline Categories & Subcategories \\
\hline
\end{tabular}

1. Insurance coverage problems

1. The lack of a systematic support system

2. High costs of rehabilitation services

3. Shortcomings in providing support services

1. The ignorance of professional ethics by therapists

2. No commitment to the observance of professional ethics

2. Unreal promises by therapists

3. Choosing profit over decent services

3. Neglecting the structure and policy of ASD rehabilitation

1. The uncertain structure of rehabilitation in Iran

2. The lack of the comprehensive rehabilitation policies

3. Inefficiency and the lack of coordination among autism associations

4. Shortcomings of rehabilitation monitoring systems

5. Abandoned teamwork concerning ASD

6. Flawed information system

1. The lack of specific protocols for the rehabilitation process

2. The lack of ASD-related protocols and evaluation methods

3. The ignorance of screening

rehabilitation protocols

4. Defects of existing rehabilitation tools

5. The confusion of families in the rehabilitation process

1. Insufficient therapists' knowledge and awareness of ASD

2. The lack of knowledge and proper attitude of families towards ASD

5. The lack of knowledge and proper attitude

3. The rejection of the child diagnosed with ASD

4. The lack of knowledge and proper attitude in the society towards ASD

1. Poor access to rehabilitation centers

2. The migration of therapists and families to metropolitan areas

6. Barriers to easy access to rehabilitation services

3. The lack of comprehensive autism rehabilitation and maintenance centers

4. Insufficient facilities in rehabilitation clinics 
high costs of rehabilitation services, and deficits in providing support services. Providing well-functioning and systematic support are critical in all healthcare centers. This is because such services need to be available throughout life for patients to go back to a normal lifestyle. Numerous individuals with ASD need to use rehabilitation services; however, they lack a high income and are unable to pay exorbitant and lifelong costs of rehabilitation services. Thus, a regular support system, like government support can highly assist these families. Moreover, insurance that covers these services can greatly improve the living condition of children with ASD. In this regard, one of the research participants mentioned: "I have registered my kid in 10-15 classes per week. It is really hard for me to afford all of them given that insurance often does not provide any support. Last year, we paid 75000 Tomans per session and this year, we are paying 95000 Tomans. The costs are very high for us and other families due to the number of classes considered for our children per month". (Interview 3)

The lack of commitment to the observance of professional ethics

The lack of commitment to professional ethics was among the barriers mentioned by numerous study participants. This category had 3 subcategories, as follows: therapists who disregard professional ethics; unreal promises by therapists, and the dominance of profitability over-provisioning service. The ignorance of ethics, in any profession, has corrupting sequences. The professional ethics and its requirements are very significant and stakes are even higher in rehabilitation professions, i.e. concerned with individuals' health. One of the study participants stated: "Some therapists guarantee their work, and this is a huge problem. We have scientific, ethical, and professional problems as well as issues in acquiring information. For example, I had a client who came to me after 3 years of massage by another therapist. Well, we did not receive such teaching and protocol at the university; no one morally approves wasting a child's time with something we are not sure about". (Interview 1)

Neglecting the structure and policy of ASD rehabilitation

Neglecting the structure and policy of rehabilitation was a major challenge mentioned by some therapists. This category included 6 subcategories, a follows flawed information system; the lack of teamwork concerning ASD; an uncertain structure of rehabilitation in Iran; the lack of comprehensive rehabilitation policies; shortcomings in rehabilitation monitoring systems, and ineffi- ciency or the lack of coordination of ASD associations Uncertainty about the overall structure was a waste of time and money for all individuals in rehabilitation. The importance of policymaking at higher levels for rehabilitation presents better healthcare systems as well as in more efficient rehabilitation teams. A study participant argued: "Rehabilitation field is usually considered with defects and no specific structure. Despite the imposed war, rehabilitation has never been considered a major issue; its weaknesses are rooted in before the victory of the revolution, as they always consider rehabilitation concerning Non-Governmental Organizations (NGOs) as a side job". (Interview 13)

\section{The lack of comprehensive ASD diagnostic and rehabilitation protocols}

One of the main concerns of most research participants was the lack of comprehensive protocols in the process of diagnosis and rehabilitation. This category included the following 5 subcategories: the lack of specific protocols in the rehabilitation process; the lack of ASD diagnosis protocols and evaluation methods; the negligence of screening; weaknesses in existing rehabilitation assessment tools, and family confusion in the rehabilitation process.

The lack of comprehensive protocols for the diagnosis and rehabilitation of ASD allows therapists to convey their personal views on the diagnosis and rehabilitation of ASDs. Anticipating and considering these protocols will increase the coordination and integration in the rehabilitation of children with ASD. One of the clinician participants mentioned this issue as: "We lack special protocols for these children to present to the family. For example, this child has such problems as, failure to understand this proverb, in these situations. He does not know what to do, in very simple cases, he is deceived and does not understand the concept of lying; well in these cases, there is no specific protocol. We do whatever comes to mind for these kids". (Interview 6)

\section{The lack of knowledge and proper attitude}

A fundamental extracted challenge was the lack of knowledge and proper attitude toward all aspects of ASD rehabilitation. This theme consisted of the following 4 subgroups: therapists' inadequate awareness of ASD; the lack of knowledge and proper attitude of families towards ASD; the lack of knowledge and attitude on the side of the society toward ASD, and the rejection of the child with a diagnosis of ASD. Increasing the level of knowledge and awareness about all aspects of ASD and its rehabilitation process modifies individuals' attitudes 
toward problems. In other words, the lower the level of individuals' literacy, the more unrealistic their expectations toward issues. The lack of knowledge and proper attitude is observed among therapists, families, and other community members; unfortunately, it minimizes the extent of understanding and acceptance of children with ASD and their rehabilitation. One of the experienced therapists stated: "The second challenge concerns associations, in which the social media must resolve it. This is a knowledge challenge. The family is misled by information, different terms, etc.; however, many of these terms, such as autism and autism spectrum, etc. are no different. Another issue is the challenge of attitude and knowledge. We must promote families' attitudes about this disorder". (Interview 10).

\section{Barriers to easy access to rehabilitation services}

The lack of easy access to services was another challenge raised in the interviews, i.e. mentioned by many of the participating parents. The lack of easy access to services included the following four sub-categories: poor access to rehabilitation centers; the migration of therapists and families to metropolitan areas; the lack of comprehensive rehabilitation and ASD care centers, and insufficient facilities for rehabilitation clinics. Despite the increase in the number of rehabilitation clinics, the lack of access to these centers seems to be due to their disproportionate distribution, which causes distant families from these centers. Furthermore, most of these rehabilitation centers are public and not specialized for children with ASD. Thus, families sometimes have to leave their hometowns and migrate to metropolitan regions to receive specialized ASD services. Regarding the access challenge, one parent stated: "Most of the clinics we access, are unsuitable for children with ASDs. They lack all the necessary experts for these kids. The family has to go to the west of Tehran for visiting the psychiatrist; to the east of Tehran for referring to the rehabilitation clinic, and to the south to visit a psychologist. In short, it would be great if centers were covering all the specialties required by these children". (Interview 8)

\section{Discussion}

The current study identified the challenges of access and use of rehabilitation services for children with ASDs and their parents. This study addressed the significant importance of rehabilitation services for this population. The relevant findings highlighted numerous factors, such as challenges in accessing and using rehabilitation services for these groups and the lack of rehabilitation efficacy in individuals affected with ASD. Ultimately, it affected the quality of life of these individuals. Furthermore, based on the study participants' perceptions and experiences (i.e. two groups of rehabilitation service providers, including therapists and the parents of children with ASD), the main category of challenges included the following.

The study participant's experiences led to the derivation of the main concept of the lack of a systematic support system. Rehabilitation insurance coverage, reduced cost of rehabilitation, and government support will lead to the optimal use of rehabilitation services in the parents of children with ASD. The existence of obstacles in any of the above mentioned disrupt the use of rehabilitation services for the family; subsequently, they are unable to use the services as much as necessary. As a result, the child presents with some anomalies. In addition to the affected child and family, the lack of a systematic support system impacts rehabilitation centers and service providers.

Pisula et al. addressed the high service costs for children with ASD [21]. Rini Vohra et al. reported insufficient insurance coverage to services for individuals with ASD [11]. Harkins et al. pointed to a lack of government support for the ASD population [22]. Ray Maker et al. argued that a major barrier to healthcare for adults with ASD is financial burdens [23]. The evidence reflected such problems as, the lack of financial resources and insufficient insurance coverage. Some participants also stated that the cost of the services their child requires is more than their income and not covered by insurance [24].

The lack of commitment to the observance of professional ethics was another obstacle, i.e. addressed by numerous participants, especially parents. In this main category, subcategories, such as false therapeutic promises of therapists; therapists' disregard for professional ethics, and the dominance of profitability over provide rehabilitation service were referred. Moreover, Abdi et al. considered those as problems associated with professional ethics in rehabilitation services [25].

One of the most fundamental challenges outlined by the study participants' understanding and the experience was mentioned in the form of the main category, called the lack of easy access to services. Numerous related studies have addressed this challenge in some manners. For example, a barrier cited by primary care providers in the Harkins study was the lack of access to ASD specialists [22]. In Ahmadi's study, parents' unawareness of accessing the required services was documented [26]. Studies signified accessibility problems, such as limitedservice providers, long waiting times, and the lack of transportation [27-29]. Areej et al. reported significant 
deficiencies in service access, including referral problems for essential services in Jeddah, Saudi Arabia [30].

A major obstacle in healthcare services accessing is the lack of comprehensive autism diagnostic and rehabilitation protocols. The lack of such protocols affects the quality of services and reduces the efficiency and effectiveness of rehabilitation services for individuals with ASD. Dadashi suggested critical challenges in providing services to individuals with ASD to concern diagnosis and intervention as well as the quality of services [31].

Among the main categories established, neglecting the structure and policy of rehabilitation was a prominent characteristic, Besides, it includes sub-categories, such as an uncertain structure and poor supervision of ASD rehabilitation; the lack of comprehensive rehabilitation policies, and inefficiency and the lack of coordination of ASD association. Some studies on the challenges of ASD rehabilitation services addressed the following points. Anderson et al. revealed some problems of the program, such as bureaucracy and unfulfilled official goals [27]. Akbarpour documented that health policymakers and managers should adopt special measures to prevent and control this disorder; accordingly, it helps to cope with difficulties in treatment, manage costs, and provide socio-economic support [32]. Abdi et al. identified the challenges of rehabilitation services for Persons With Disabilities (PWDs). They also highlighted the important role of teamwork and therapists in rehabilitation services [33]. Another article also signified the poor cooperation of parents and professionals in the planning, implementation, and evaluation of medical services; the heterogeneity of services provided in different centers was also addressed [26].

One of the main categories related to the challenges of accessing and using rehabilitation services was the poverty of knowledge and attitude, i.e. consistent with previous studies. For example, Harkins cited the lack of prior education on ASD and the lack of knowledge about the sources of the disorder as barriers to ASD [22]. Nicolaidis et al. referred to labeling ASD [10]. Evidence demonstrated a lack of understanding and knowledge in this respect [24, 34]. Moreover, Tekola et al. suggested that lack of awareness and labeling is an obstacle to the proper care of these children [35].

A limitation of this study was the impossibility of conducting face-to-face interviews with all study participants; some interviews were performed online using the social networks, such as WhatsApp, due to the current Covid-19 pandemic. Another limitation of this study was the lack of interviews with other stakeholders, including policymakers, intervention managers, and ASD associations, which could be beneficial in future studies.

\section{Conclusion}

The present study signified numerous challenges in children, families, and therapists in accessing and using ASD-concerned rehabilitation services. Such shortcomings are especially observed in developing countries, like Iran, where adequate financial support and medical services are not provided. Besides, lifelong medical and rehabilitation services are not planned for children with ASD. In other words, there is a serious need for comprehensive treatment and rehabilitation planning for children with ASD. Culture and education in society should be established from childhood respecting public education; they should also be developed in school courses in more specialized fields.

Furthermore, policymakers and service providers must be aware of the challenges of rehabilitation services for individuals with ASD and pay special attention to this issue. Accordingly, further qualitative studies are recommended in this respect. The obtained results reflected that due to the emergence of serious problems in the access and use of rehabilitation services for ASD and their families, it is necessary to adopt fundamental measures to solve these problems. Significant modifications should also be made in this regard.

It is expected that this study provides evidence for policymakers and intervention managers in accessing and using rehabilitation services for children with ASD. Such measures could help to improve their received services and living conditions; ultimately, it leads to improved quality of life in this population.

\section{Ethical Considerations}

\section{Compliance with ethical guidelines}

This study was approved by the ethics committee of the University of Social Welfare and Rehabilitation Sciences (Code: IR.USWR.REC.1399.144).

\section{Funding}

This research did not receive any grant from funding agencies in the public, commercial, or non-profit sectors. 


\section{Authors' contributions}

Conceptualization, Investigation, Writing - original draft: Mahta Alsadat Aarabi, Kianoush Abdi; Data collection: Mahta Alsadat Aarabi; Methodology \& editing: Mahta Alsadat Aarabi, Kianoush Abdi; Data analysis: All authors; Supervision: Kianoush Abdi and Mohammad Saeed Khanjani.

\section{Conflict of interest}

The authors declared no conflicts of interest.

\section{Acknowledgments}

The authors would like to thank all participants who contributed to this research

\section{References}

[1] Hodges H, Fealko C, Soares N. Autism Spectrum Disorder: Definition, epidemiology, causes, and clinical evaluation. Translational Pediatrics. 2020; 9(suppl 1):S55-s65. [DOI:10.21037/tp.2019.09.09] [PMID] [PMCID]

[2] American Psychiatric Association. Diagnostic and statistical manual of mental disorders (DSM-5®). Washington, D.C.: American Psychiatric Pub; 2013.

[3] Aarabi MA, Abdi K, Khanjani MS. Letter to editor: COVID-19 and mental health of people with Autism Spectrum Disorder and their families; what can be done? Middle East Journal of Rehabilitation and Health Studies. 2021; 8(2):e112188. [DOI:10.5812/mejrh.112188]

[4] Knopf A. Autism prevalence increases from 1 in 60 to 1 in 54 CDC. The Brown University Child and Adolescent Behavior Letter. 2020; 36(6):4. [DOI:10.1002/cbl.30301]

[5] Li HJ, Chen CY, Tsai CH, Kuo CC, Chen KH, Chen KH, et al. Utilization and medical costs of outpatient rehabilitation among children with autism spectrum conditions in Taiwan. BMC Health Services Research. 2019; 19(1):354. [DOI:10.1186/ s12913-019-4193-z] [PMID] [PMCID]

[6] Golestan S, Soleiman P, Moradi H. Feasibility of using sphero in rehabilitation of children with autism in social and communication skills. Paper presented at: International Conference on Rehabilitation Robotics (ICORR). 17-20 Jul 2017, London, UK. [DOI:10.1109/ICORR.2017.8009378] [PMID]

[7] Sengupta K, Lobo L, Krishnamurthy V. Educational and behavioral interventions in management of Autism Spectrum Disorder. Indian Journal of Pediatrics. 2017; 84(1):61-7. [DOI:10.1007/s12098-015-1967-0] [PMID]

[8] Dern S, Sappok T. Barriers to healthcare for people on the autism spectrum. Advances in Autism. 2016; 2(1). [DOI:10.1108/ AIA-10-2015-0020]
[9] Nicolaidis C, Raymaker D, McDonald K, Dern S, Boisclair WC, Ashkenazy E, et al. Comparison of healthcare experiences in autistic and non-autistic adults: A cross-sectional online survey facilitated by an academic-community partnership.Journal of General Internal Medicine. 2013; 28(6):761-9. [DOI:10.1007/s11606-012-2262-7] [PMID] [PMCID]

[10] Nicolaidis C, Raymaker DM, Ashkenazy E, McDonald KE, Dern S, Baggs AE, et al. "Respect the way I need to communicate with you": Healthcare experiences of adults on the autism spectrum. Autism. 2015; 19(7):824-31. [DOI:10.1177/1362361315576221] [PMID] [PMCID]

[11] Vohra R, Madhavan S, Sambamoorthi U, St Peter C. Access to services, quality of care, and family impact for children with autism, other developmental disabilities, and other mental health conditions. Autism. 2014; 18(7):815-26. [DOI:10.1177/1362361313512902] [PMID] [PMCID]

[12] Aarabi M, Abdi K, Khanjani M. Using of rehabilitation services, the main concern of Autism Spectrum Disorder: What can be done? Journal of Rehabilitation Sciences \& Research. 2020; 7(4):189-90. https://jrsr.sums.ac.ir/article_47289_40def 5e07c420efe8b5d67b207a0bf86.pdf

[13] Zerbo O, Massolo ML, Qian Y, Croen LA. A study of physician knowledge and experience with autism in adults in a large integrated healthcare system. Journal of Autism and Developmental Disorders. 2015; 45(12):4002-14. [DOI:10.1007/ s10803-015-2579-2] [PMID]

[14] Valentine K. A consideration of medicalisation: Choice, engagement and other responsibilities of parents of children with autism spectrum disorder. Social Science \& Medicine. 2010; 71(5):950-7. [DOI:10.1016/j.socscimed.2010.06.010] [PMID]

[15] Kondracki NL, Wellman NS, Amundson DR. Content analysis: Review of methods and their applications in nutrition education. Journal of Nutrition Education and Behavior. 2002; 34(4):224-30. [DOI:10.1016/S1499-4046(06)60097-3]

[16] Hsieh HF, Shannon SE. Three approaches to qualitative content analysis. Qualitative Health Research. 2005; 15(9):1277-88. [DOI:10.1177/1049732305276687] [PMID]

[17] Graneheim UH, Lundman B. Qualitative content analysis in nursing research: Concepts, procedures and measures to achieve trustworthiness. Nurse Education Today. 2004; 24(2):105-12. [DOI:10.1016/j.nedt.2003.10.001] [PMID]

[18] Elo S, Kyngäs H. The qualitative content analysis process. Journal of Advanced Nursing. 2008; 62(1):107-15. [DOI:10.1111/j.1365-2648.2007.04569.x] [PMID]

[19] Guba EG. Criteria for assessing the trustworthiness of naturalistic inquiries. Ectj. 1981; 29(2):75-91. https:/ /link.springer.com/article/10.1007/BF02766777

[20] Baptiste I. Qualitative data analysis: Common phases, strategic differences. Qualitative Methods in Various Disciplines II: Cultural Sciences. 2001; 2(3). [DOI:10.17169/fqs-2.3.917]

[21] Płatos M, Pisula E. Service use, unmet needs, and barriers to services among adolescents and young adults with autism spectrum disorder in Poland. BMC Health Services Research. 2019, 19(1):587. [DOI:10.1186/s12913-019-4432-3] [PMID] [PMCID]

[22] Mazurek MO, Harkins C, Menezes M, Chan J, Parker RA, Kuhlthau K, et al. Primary Care Providers' Perceived Barriers and Needs for Support in Caring for Children with Autism. 
The Journal of Pediatrics. 2020; 221:240-5.e1. [DOI:10.1016/j. jpeds.2020.01.014] [PMID]

[23] Raymaker DM, McDonald KE, Ashkenazy E, Gerrity M, Baggs AM, Kripke C, et al. Barriers to healthcare: Instrument development and comparison between autistic adults and adults with and without other disabilities. Autism. 2017; 21(8):972-84 [DOI:10.1177/1362361316661261] [PMID] [PMCID]

[24] Jones AP. Caregivers' Challenges in Accessing Services for Children with Autism. 2019. https://scholar.google.com/ scholar?hl=en\&as_sdt $=0 \% 2 \mathrm{C} 5 \& \mathrm{q}=+$ Jones $+\mathrm{AP} .+$ Caregivers $\%$ E2\%80\%99+Challenges+in+Accessing+Services+for+Childre n+with+Autism.+2019.\&btnG=

[25] Abdi K, Arab M, Khankeh HR, Kamali M, Rashidian A, Farahani FK, et al. Challenges in providing rehabilitation services for people with disabilities in Iran: A qualitative study. Journal of Advances in Medicine and Medical Research. 2016; 13(4):1-11. [DOI:10.9734/BJMMR/2016/23337]

[26] Ahmadi A, Zalani HA, Amrai K. The needs of Iranian families of children with autism spectrum disorder, cross-cultural study. Procedia-Social and Behavioral Sciences. 2011; 15:3216. [DOI:10.1016/j.sbspro.2011.03.094]

[27] Anderson C, Lupfer A, Shattuck PT. Barriers to receipt of services for young adults with Autism. Pediatrics. 2018 141(Suppl 4):S300-s5. [DOI:10.1542/peds.2016-4300G] [PMID]

[28] Rabanifar N, Abdi K. Letter to Editor: Telerehabilitation: A Useful and appropriate approach for people with disability in Covid-19 pandemic. Medical Journal of The Islamic Republic of Iran (MJIRI). 2021; 35(1):125-6. http://mjiri.iums.ac.ir/ article-1-7293-en.pdf

[29] Rabanifar N, Abdi K. Rehabilitation services: Why should we use tele-rehabilitation in Iran? Necessity and application. Iranian Rehabilitation Journal. 2019; 17(4):293-6. [DOI:10.32598/irj.17.4.293]

[30] Babatin AM, Alzahrani BS, Jan FM, Alkarimi EH, Jan MM. The availability of services for children with autism spectrum disorder in a Saudi population. Neurosciences (Riyadh, Saudi Arabia). 2016; 21(3):223-6. [DOI:10.17712/nsj.2016.3.20150597] [PMID] [PMCID]

[31] Dadashi Z. Exploring the expectations and experiences of service providers in the services provision for children with Autism Spectrum Disorder in Tabriz (Persian)] [MA thesis]. Tabriz: Tabriz University of Medical Sciences and Health Services Faculty of management and medical informatics; 2019. http://dspace.tbzmed.ac.ir:8080/xmlui/handle/123456789/60747

[32] Mosadeghrad AM, Pour-reza A, Akbarpour N. [Determinants of economic burden of autism (Persian)]. 2018 76(10):665-71. https://tumj.tums.ac.ir/browse.php?a id $=9278 \&$ sid $=1 \&$ slc $\_$lang $=$en

[33] Abdi K, Arab M, Rashidian A, Kamali M, Khankeh HR, Farahani FK. Exploring barriers of the health system to rehabilitation services for people with disabilities in Iran: A qualitative study. Electronic Physician. 2015; 7(7):1476-85. [DOI:10.19082/1476] [PMID] [PMCID]

[34] Najafi Z, Abdi K, Khanjani MS, Dalvand H, Amiri M. Convention on the rights of persons with disabilities: Qualitative exploration of barriers to the implementation of articles 25 (health) and 26 (rehabilitation) in Iran. Medical Journal of The
Islamic Republic of Iran (MJIRI). 2021; 35(1):78-86. http:// mjiri.iums.ac.ir/article-1-6435-en.pdf

[35] Tekola B, Baheretibeb Y, Roth I, Tilahun D, Fekadu A, Hanlon $\mathrm{C}$, et al. Challenges and opportunities to improve autism services in low-income countries: lessons from a situational analysis in Ethiopia. Glob Ment Health (Camb). 2016; 3:e21. [DOI:10.1017/gmh.2016.17] [PMID] [PMCID] 